\title{
Smart food...vegetables may be smarter than we think: A word about multisite near-infrared spectroscopy
}

\author{
Rohit Rao, MD, MBA, ${ }^{\mathrm{a}}$ and Tara Karamlou, MD, $\mathrm{MSc}^{\mathrm{b}}$
}

\footnotetext{
From the ${ }^{a}$ Division of Cardiology, Department of Pediatrics, University of California San Diego and Rady Children's Hospital, San Diego, Calif; and ${ }^{\mathrm{b}}$ Division of Pediatric Cardiac Surgery, Department of Surgery, Western Regional Medical Center and Rady Children's Hospital, San Diego, Calif.

Disclosures: Authors have nothing to disclose with regard to commercial support.

Received for publication Aug 22, 2018; accepted for publication Aug 24, 2018; available ahead of print Sept 25, 2018.

Address for reprints: Rohit Rao, MD, MBA, Department of Pediatrics, Rady Children's Hospital San Diego, UC San Diego School of Medicine, 3020 Children's Way, San Diego, CA 92123 (E-mail: RRao1@ @chsd.org). J Thorac Cardiovasc Surg 2018;156:2258-9

$0022-5223 / \$ 36.00$

Copyright (c) 2018 Published by Elsevier Inc. on behalf of The American Association for Thoracic Surgery https://doi.org/10.1016/j.jtcvs.2018.08.048
}

Kahn and Anyanwu ${ }^{1}$ recently published an observational nonblinded study comparing cerebral near-infrared spectroscopy (NIRS) values of cardiothoracic surgeons and anesthesiologists with vegetables: yams, courgettes, and butternut squash. They reported that the butternut squash had NIRS values similar to the human brains in their population. ${ }^{1}$ Smart food generally refers to foods such as blueberries and leafy greens, which confer neuroprotection by scavenging free radicals. However, the study by Kahn and Anyanwu ${ }^{1}$ is a somewhat whimsical reminder that we must be wary that diagnostic testing may sometimes lack clinical correlation. In this issue of the Journal, Koichi and colleagues $^{2}$ have published their results of NIRS monitoring of the thigh for monitoring the adequacy of high-flow regional cerebral perfusion (HFRCP). A unique feature of this study is the choice of the thigh as one of the NIRS monitoring sites.

Smart vegetables aside, the current evidence of NIRS monitoring in human studies over the past decade is consistent with class II, level $\mathrm{B}$ recommendations according to the American Heart Association and the American College of Cardiology Task Force on Practice Guidelines. ${ }^{3}$

Koichi and colleagues ${ }^{2}$ report the results of a prospective cohort study of 20 consecutive patients undergoing aortic arch repair at their institution between 2009 and 2016 . The objective of the study was to evaluate markers of peripheral perfusion during high-flow regional cerebral perfusion. To this end, the authors used NIRS to measure regional oxygen saturation on the forehead, trunk, and thigh. In addition, they calculated the oxygen delivery ratio (defined as the quotient of oxygen delivery [DO2] during HFRCP divided by DO2 before HFRCP) and correlated these metrics with other clinical variables. The authors found, not surprisingly, that the forehead NIRS values increased during a period of descending aortic clamping, whereas the thigh NIRS values decreased during this period. Further, accepted markers of perfusion, such as

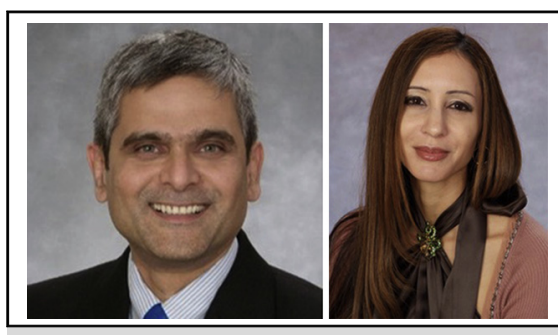

Rohit Rao, MD, MBA, and Tara Karamlou, MD, MSc

Central Message

Multisite NIRS monitoring is a tool to measure perfusion because it exploits the differential regulation of competing tissue beds. However, the optimum somatic sites for ascertainment remain unclear

See Article page 2251 .

postoperative creatinine kinase (CK) and lactate levels, were associated on multivariable or correlational analysis with prolonged cardiopulmonary bypass times, nadir thigh regional oxygen saturation ( $\mathrm{rSO} 2)$, and $\mathrm{DO} 2$ ratio. The receiver operating characteristic analysis demonstrated a possible threshold of less than 0.66 for the DO2 ratio for discriminating high lactate levels greater than $5 \mathrm{mmol} / \mathrm{L}$.

This is an important study and presents an opportunity to elucidate the concept that an optimum measure of the adequacy of perfusion involves integration of multiple regional sites simultaneously, mainly because of the differential regulation and sensitivity of regional tissue beds in the body. Recent articles that have used multisite NIRS monitoring have focused attention on this concept, ${ }^{4}$ and the authors of this study contribute to the advancement and elucidation of these principles. NIRS devices measure the venous weighted oxyhemoglobin saturation in a field of tissue, rather than in arteries, and thus the $\mathrm{rSO} 2$ parameter provides a window into regional oxygen supply-demand relationships. Blood flow distribution during HFRCP depends on the interaction of various regional centric flow-metabolism coupling relationships that affect local vascular resistances. Thus, monitoring the rSO2 of multiple organ tissue beds under different physiologic control during HFRCP has the potential to provide real-time flow-metabolism coupling dynamics in vascular beds with differing physiologic control mechanisms. The ability to monitor multiple sites is perhaps 
even more important during the perioperative setting as variations in regional blood flow patterns are likely to emerge under different physiologic conditions. Brain and kidney monitoring represent 2 poles of circulatory hierarchies, in which one is a highly autoregulated, high extraction site and the other is a low extraction site with heavy sympathetic innervation. On the contrary, the thigh $\mathrm{rSO} 2$ is a marker of flow to an organ with low fixed oxygen extraction under conditions of anesthesia and neuromuscular blockade. Under these circumstances, it is not surprising that the $\mathrm{rSO} 2$ of the thigh was higher than the renal site. The renal bed will have additional blood flow variability based on the sympathetic tone activation in response to a low flow state. Given this, it is plausible that the thigh bed might be a better marker of lower DO2 during HFRCP because these data isolate flow per se because of the absence of much sympathetic innervation and a stable oxygen consumption state.

The authors have correlated the thigh NIRS data and DO2 ratios with both $\mathrm{CK}$ levels and lactate levels. Although both of these (CK less so) are intuitively associated with malperfusion, the authors have presented no clinical data that the outcomes among those with higher CK or lactate levels had worse outcomes, including length of stay, major morbidity, or mortality. At the risk of sounding contrarian, the values of specific markers, whether biomarkers or NIRS, must have meaningful clinical outcome correlates.

The study by Koichi and colleagues ${ }^{2}$ is yet another example of the versatility and utility of NIRS data and, as mentioned earlier, a reminder that the physiologic correlates of NIRS data are complex and incompletely understood. Thus, the article by Kahn and Anyanwu ${ }^{1}$ highlighting the limitations of NIRS is timely in the present day. It reminds us that we must be responsible stewards of the ways we interpret and integrate NIRS data into clinical practice.

In the end, we wondered which vegetable would be closer to the thigh NIRS? We are partial to broccoli.

\section{References}

1. Kahn RA, Anyanwu A. Near-infrared spectroscopy in vegetables and humans: an observational study. Eur J Anaesthesiol. July 16, 2018 [Epub ahead of print].

2. Sughimoto K, Kohira S, Hayashi H, Torii S, Kitamura T, Horai T, Miyaji K Markers of peripheral perfusion during high-flow regional cerebral perfusion for aortic arch repair. J Thorac Cardiovasc Surg. 2018;156:2251-7.

3. Ghanayem NS, Wernovsky G, Hoffman GM. Near-infrared spectroscopy as a hemodynamic monitor in critical illness. Pediatr Crit Care Med. 2011; 12(4 Suppl):S27-32

4. Hoffman GM, Ghanayem NS, Scott JP, Tweddell JS, Mitchell ME, Mussatto KA. Postoperative cerebral and somatic near-infrared spectroscopy saturations and outcome in hypoplastic left heart syndrome. Ann Thorac Surg. 2017;103:1527-35. 